\section{Molecular subtypes of gastrointestinal stromal tumors and their prognostic and therapeutic implications}

\author{
Zoltan Szucs', Khin Thway', Cyril Fisher', Ramesh Bulusu², Anastasia Constantinidou', \\ Charlotte Benson', Winette TA van der Graaf ${ }^{1,3}$ \& Robin L Jones ${ }^{*, 1}$
}

\begin{abstract}
Gastrointestinal stromal tumors (GISTs) are composed of various molecular subtypes, with differing prognostic and predictive relevance. Previously, tumors lacking mutations in the KIT and PDGFRA genes have been designated as 'wild-type' GISTs; however, they represent a heterogeneous group currently undergoing further subclassification. Primary and secondary resistance to imatinib poses a significant clinical challenge, therefore ongoing research is trying to evaluate mechanisms to overcome resistance. Thorough understanding of the prognostic and predictive relevance of different genetic subtypes of GIST can guide clinical decision-making both in the adjuvant and the metastatic setting. Further work is required to identify tailored therapies for specific subgroups of GISTs wild-type for KIT and PDGFRA mutations and to identify predictive factors of resistance to currently approved systemic therapies.
\end{abstract}

First draft submitted: 14 April 2016; Accepted for publication: 29 July 2016; Published online: 7 September 2016

Gastrointestinal stromal tumors (GISTs) are the most common mesenchymal tumors of the digestive tract, representing $0.1-3 \%$ of all GI cancers. GISTs can arise from any part of the GI tract, primarily within the muscular wall of the stomach and small intestine and rarely in extra-intestinal locations (omentum, mesentery, retroperitoneum or pelvic cavity) [1]. The cells of origin of GISTs are thought to be the interstitial cells of Cajal or their precursors [2]. GISTs represent a wide spectrum of disease, with aggressiveness of the disease correlating with tumor size, mitotic activity and anatomical origin (these three clinicopathological features forming the basis of currently used risk-stratification systems) $[1,3-5]$.

Surgery is the mainstay of management for localized GIST and is curative in $45-60 \%$ of cases $[3,4]$. Locally advanced or metastatic GISTs are notoriously refractory to conventional chemotherapy or radiation. The discovery of the KIT tyrosine kinase receptor and subsequently that of the mutually exclusive KIT and PDGFRA gain of function mutations have provided a paradigm shift in the way we classify, diagnose and most importantly treat GISTs. Studies of KIT/PDGRA mutation negative or 'wild-type' (WT) GISTs have uncovered numerous other molecular groups, including mutations in $B R A F$ and subunits of the succinate dehydrogenase $(\mathrm{SDH})$ complex. Routine genotyping has become an integral part of management of GISTs undergoing tyrosine kinase inhibitor (TKI) therapy [5].

The objective of this manuscript is to mirror the evolution of GIST subclassification based on genetic profiling and to highlight the distinct prognostic and predictive relevance of already

\section{KEYWORDS}

- BRAF・GIST • KIT

- molecular subgroups

- NF-1 • PFGFRA • SDH

deficiency • 'wild-type' GIST 
well-characterized and yet emerging genetic alterations in GIST. We separate in our discussion the prognostic and predictive relevance of specific genetic subtypes of GIST, therefore providing a more transparent guide for clinical decision-making in both the adjuvant and metastatic setting.

\section{The KIT receptor tyrosine kinase}

The $c$-kit proto-oncogene is allelic with the murine white-spotting locus (W), mutations of which affect melanogenesis, gametogenesis and hematopoiesis during development and in adult life [6]. $c$-kit encodes the $145-\mathrm{kDA}$ receptor tyrosine kinase (RTK) KIT and is the normal cellular homolog of the viral oncogene $v$-kit, the transforming gene of the Hardy-Zuckermann 4 feline sarcoma virus [7]. KIT is a member of the type III RTK family which includes the PDGFRA and PDGFRB, the macrophage colony-stimulating-factor receptor (CSF1R) and the Fl cytokine receptor (FLT3) [8]. The CD117 antibody against KIT has previously been shown to be a sensitive and specific marker for GIST, being positive in $95 \%$ of GIST specimens [9].

The KIT transmembrane receptor is composed of an extracellular domain consisting of five immunoglobulin (Ig) like motifs, a transmembrane hinge, a juxta-membrane (JM) domain and an intracellular tyrosine kinase domain consisting of two regions separated by a kinase insert domain (KID). The different segments of the KIT receptor all have a specific designated role in the process of tyrosine kinase activation (Table 1 ).

In around $82-87 \%$ of all cases, GISTs have activating mutations in either the KIT or the homologous PDGFRA RTKs (Table 2). Gain of function mutation of either KIT or PDGRA receptors lead to constitutive, ligandindependent activation that results in the activation of Ras/Raf/MAPK, JAK/STAT3 and PI3K/Akt/mTOR downstream pathways, ultimately increasing cell proliferation and inhibiting apoptosis $[10,17]$.

\section{- KIT exon 9, 11, 13, 17 mutant GIST}

While most KIT mutations in GIST are somatic, germline mutations have been identified in a small number of families [5]. Gain-of-function mutations in KIT result in growth advantage by constitutive, ligand-independent activation of the RTK [18]. While most GISTs are heterozygous for a given mutation, in around 15\% of tumors the remaining WT KIT allele is lost and this is associated with malignant behavior, increased mitotic activity and topoisomerase II expression [19].

Approximately $69-83 \%$ of all GISTs show a KIT mutation (Table 2). An important observation is that KIT detection by immunohistochemistry (IHC) is unrelated to the existence of underlying mutations. The vast majority of $K I T$ ' 'hot spot' mutations are found in exon 11, less frequently in exon 9 and rarely in exon 13 and exon 17 (Table 2) [20]. Primary nonhot spot exon 8 mutation of the KIT receptor is extremely rare and is not routinely screened for (Table 2) [11].

\section{- KIT exon 11 mutational landscape}

The most common site of KIT mutation is in the 5 ' end of exon 11, which encodes the JM domain, and in the overwhelming majority of cases deletions or substitutions involving codons 550-560 occur [12]. Genetic alterations in exon 11 disrupt the auto-inhibitory function and trigger ligand-independent receptor activation [21].

There is a constantly growing body of evidence that the exact types of genetic alterations hold strong clinical prognostic value of their own [13,14]. Wozniak and colleagues analyzed clinical follow-up data of 427 patients who

\section{Table 1. KIT receptor function and primary mutational status in gastrointestinal stromal tumors.}

\begin{tabular}{|c|c|c|c|c|}
\hline $\begin{array}{l}\text { Coding } \\
\text { region }\end{array}$ & $\begin{array}{l}\text { KIT } \\
\text { segment }\end{array}$ & Physiological function & $\begin{array}{l}\text { Primary } \\
\text { mutation rate }\end{array}$ & Ref. \\
\hline Exon 8 & ECIGM & SCF/ligand binding; dimerization domain & $0.15-0.23 \%$ & {$[10,11]$} \\
\hline Exon 9 & ECIGM & $\begin{array}{l}\text { SCF/ligand binding triggering subsequent receptor homodimerization, conformational } \\
\text { change and kinase activation }\end{array}$ & $7-15 \%$ & {$[10,12-13]$} \\
\hline Exon 11 & JM & Auto-inhibition of the receptor in ligand-free state & $61-71 \%$ & {$[10,12,14]$} \\
\hline Exon 13 & PKD & ATP-binding region & $0.5-1.8 \%$ & {$[10,12,14]$} \\
\hline Exon 14 & KID & Undefined function beyond linking PKD with the DKD & NDA & [10] \\
\hline Exon 17 & DKD & Contains the activation loop that stabilizes the activated receptor & $0.5-1 \%$ & {$[10,12,15]$} \\
\hline
\end{tabular}

NDA: No data available as primary mutation [10-16].

DKD: Distant kinase domain; EC IGM: Extracellular immunoglobulin-like motif; JM: Juxta-membrane domain; PKD: Proximal kinase domain; SCF: Stem cell factor. 
Table 2. KIT and PDGFRA 'hot spot' mutation landscape.

\begin{tabular}{|c|c|c|c|c|c|c|c|c|c|c|c|c|}
\hline \multirow[t]{2}{*}{ Study (type) } & \multirow{2}{*}{$\begin{array}{l}\text { OMR } \\
\text { (\%) }\end{array}$} & \multicolumn{5}{|c|}{ KIT (exon); \% } & \multicolumn{5}{|c|}{ PDGFRA (exon); \% } & \multirow[t]{2}{*}{ Ref. } \\
\hline & & All & 9 & 11 & 13 & 17 & All & 18 & $18^{*}$ & 14 & 12 & \\
\hline Polish (registry) & 82.2 & 69.3 & 7.3 & 61.1 & 0.5 & 0.5 & 12.9 & 11.9 & 8.2 & 0.7 & 0.2 & [12] \\
\hline EORTC 62005 (Phase III trial) & 86.2 & 83.6 & 15.4 & 65.8 & 1.6 & 0.8 & 1.6 & NS & 1 & NS & NS & [13] \\
\hline CALGB 150105 (Phase III trial) & 84.6 & 81.9 & 8.2 & 71.3 & 1.2 & 1.0 & 2.65 & 1.2 & 0.9 & NS & 0.23 & [15] \\
\hline ACOSOG Z9001 (Phase III trial) & 87.4 & 76.2 & 6.9 & 67.3 & 1.8 & 0.2 & 11.2 & NS & 5.3 & NS & NS & [16] \\
\hline
\end{tabular}

underwent curative resection for GIST in Poland between 1999 and 2009. Surgical specimens of imatinib-naive GISTs from this period were prospectively included in the Polish Clinical GIST Registry. Mutational analysis was retrospectively carried out assessing for exon 9, 11, 13, 17 KIT and exon 12, 14, 18 PDGFRA mutational status [14]. The European ConticaGIST database study analyzed the clinicopathologic and molecular data (exon 9, 11, 13, 17 KIT and exon 12, 14, 18 PDGFRA) of 1,056 GIST patients undergoing curative R0/R1 resection. Patients were diagnosed between January 1985 and April 2012 with 13 contributing institutions from four European countries. As a strength of the ConticaGIST series, the majority of the cases (all those diagnosed from 2001) were studied prospectively (83\% of all) [13]. None of the patients in either studies were exposed to chemotherapy or any other anticancer agent, including imatinib, thus both studies provide invaluable prognostic information on the clinical course of GIST according to specific tumor genotype.

\section{Exon 11 deletions}

Deletions affecting codons 557-558 of exon 11 of the $c$-KIT gene are detected in $23.2-27.7 \%$ of all GIST cases. They are lost either as specific isolated p.W557_K558 deletions in 6.3-7.5\% of GISTs or as part of larger deletions in $15.7-21.4 \%$ of the cases (Table 3 ). Early studies associated deletions affecting codons 557-558 with an aggressive, metastasizing phenotype and indicate an overall poor prognosis [22,23] Interestingly Martin-Broto and colleagues demonstrated that the predictive value of deletion of 557/558 for recurrence might be limited only to the first 4 years after curative surgery [24]. In the Polish registry study $557 / 558$ codon deletions were more frequent in larger $(88 \%,>5 \mathrm{~cm})$ GISTs with higher mitotic index (MI; 75\% with > 5/50 HPF) and thus 80\% of them stratified as high-risk tumors. Patients with 557/558 codon deletions had a lower $23.8 \%$ 5-year relapse-free survival (RFS) rate as compared with patients with any other KIT exon 11 mutations (41.8\% RFS), but also with other exon 11 deletions that have not involved codons 557/558 (33.3\% RFS) [14].

In the Multinational European ConticaGIST Database analysis KIT p.W557_K558del mutants equally segregated in gastric and nongastric sites (55 vs 45\%). KIT p.W557_K558del was more frequently identified in patients younger than 60 years of age ( 59 vs $42.4 \%$ ), in tumors' $>5 \mathrm{~cm}$ (84.5 vs $57.7 \%$ ), with $\mathrm{MI}>5 / 50 \mathrm{HPF}$ (68.9 vs $39.4 \%$ ), and classified as high risk (70.2 vs $38.9 \%$ ), when compared with other KIT exon 11 mutated tumors. It was an important observation that the clinicopathologic characteristics of tumors bearing KIT p.W557_K558del were comparable with the group of tumors with KIT delinc557/558, within which tumor size, mitotic rate and fraction of high-risk tumors were also significantly higher than in tumors with other KIT exon 11 mutants. KIT delinc557/558 was associated with an increased risk for tumor progression with a hazard ratio (HR) of 1.45 and an inferior disease-free survival (DFS; median DFS 45.5 months; 5-year DFS 33.1\%). The relatively high number of KIT del-inc557/558 mutants equally distributed in gastric and nongastric sites enabled the researchers to analyze the possible impact of this genotype on DFS, depending on the anatomical site of GISTs. In clear contrast with other KIT exon 11, KIT exon 9 and PDGFRA exon 18 mutations, the poor prognostic impact of KIT del-inc557/558 on patients' survival was only significant in GIST localized to the stomach ( $p<0.001)$, but not in tumors' with nongastric origin $(\mathrm{p}<0.26)$. The same associations were also evident when comparing $K I T$ del-inc557/558 mutants with other exon11 KIT mutations, overall ( $\mathrm{p}<0.0001$; or comparing gastric $(\mathrm{p}<0.0001)$ and nongastric $(\mathrm{p}<0.599)$ GIST. This phenomenon might be related to the observation that gastric GIST with KIT del-inc557/558 had a larger size (7 vs $5.8 \mathrm{~cm})$ and a higher mitotic rate ( 6 vs $4 / 50 \mathrm{HPF}$ ) when 
Table 3. Exon 11 mutations and their prognostic implications.

\begin{tabular}{|c|c|c|c|}
\hline Genetic alteration & Study/mutation frequency & Clinical-pathological prognostic features & Ref. \\
\hline p.W557_K558 deletion & $\begin{array}{l}\text { Polish Registry } 7.5 \% \\
\text { ConticaGIST } 6.3 \% \\
\text { Norwegian Registry } 5.3 \% \\
\text { EORTC } 620056.9 \%\end{array}$ & $\begin{array}{l}\text { High-risk tumors } \\
\text { Higher Ml, larger ( }>5 \mathrm{~cm} \text { ) size } \\
\text { Gastric: nongastric location } 1: 1 \\
\text { Younger age at presentation ( }<60 \text { years) } \\
\text { Lower RFS rate compared with all other (KIT exon } 9 \text { and PDGFRA exon 18) } \\
\text { and to other exon } 11 \text { mutations } \\
\text { Prognostic power seems to be confined to gastric location of GISTs }\end{array}$ & {$[12-15,25]$} \\
\hline KITdelinc557/558 & $\begin{array}{l}\text { Polish Registry } 15.7 \% \\
\text { ConticaGIST } 21.4 \%\end{array}$ & $\begin{array}{l}\text { High-risk tumors } \\
\text { Higher MI, larger }(>5 \mathrm{~cm} \text { ) size } \\
\text { Gastric: nongastric location 1:1 } \\
\text { Younger age at presentation ( }<60 \text { years) } \\
\text { Lower RFS rate compared with all other (KIT exon } 9 \text { and PDGFRA exon 18) } \\
\text { and to other exon } 11 \text { mutations } \\
\text { Prognostic power seems to be confined to gastric location of GISTs }\end{array}$ & {$[12-15,25]$} \\
\hline $\begin{array}{l}\text { Intron } 10 / \text { exon } 11 \text { junction } \\
\text { deletions (resulting in } \\
\text { p.K550_K558 deletion) }\end{array}$ & Polish Registry $1.4 \%$ & $\begin{array}{l}\text { High-risk tumors } \\
\text { Presumed aggressive/metastasizing clinical behavior }\end{array}$ & [14] \\
\hline $\begin{array}{l}\text { Single nucleotide } \\
\text { substitutions }\end{array}$ & $\begin{array}{l}\text { Polish Registry } 15.5 \% \\
\text { ConticaGIST } 19.7 \% \\
\text { CALGB } 1501051.6 \% \\
\text { EORTC } 620051.8 \%\end{array}$ & $\begin{array}{l}\text { Lower Ml, smaller }(<5 \mathrm{~cm}) \text { size } \\
\text { Indolent clinical course } \\
\text { Better } 5 \text {-year RFS (as compared with KIT deletions or duplications) }\end{array}$ & {$[12-15]$} \\
\hline Duplications & $\begin{array}{l}\text { Polish Registry } 7 \% \\
\text { ConticaGIST } 6.6 \% \\
\text { EORTC } 620051.5 \%\end{array}$ & $\begin{array}{l}\text { Exclusive gastric location } \\
\text { Benign clinical outcome }\end{array}$ & {$[12-14]$} \\
\hline $\begin{array}{l}\text { Homo/hemizygous KIT } \\
\text { exon } 11 \text { mutant }\end{array}$ & Polish Registry 4\% & $\begin{array}{l}\text { High risk of early metastatic disease } \\
\text { Disseminated malignancy at presentation }\end{array}$ & [14] \\
\hline
\end{tabular}

compared with other KIT exon 11 mutants. Consequently, the fraction of patients with gastric GIST harboring KIT del-inc557/558 who relapsed 5 years after surgery was twice as high as patients with other KIT exon 11 mutations (61 vs 29\%). Even in tumors classified as nonhigh risk ([very]low and intermediate) and originating from the stomach, the presence of KIT del-inc557/558 remained an important prognosticator for poor outcome in comparison with other KIT exon 11 mutations, KIT exon 9 and PDGFRA exon 18 mutations [13].

\section{Intron 10/exon 11 junction deletions}

In the Polish registry series six GISTs $(1.4 \%$ of all) had deletions affecting the intron 10 /exon 11 junction, resulting in p.K550_K558 deletion as such. Among these handful GISTs all but one tumor was high risk or overtly malignant at presentation, suggesting a more aggressive clinical behavior of tumors with such deletion [14].

KIT exon 11 single nucleotide substitutions A discrepancy in the frequency of KIT exon 11 substitutions between population-based studies
$15.5-28.6 \%$ and tumor material from clinical trials $1.6-1.86 \%$ has been observed (Table 3 ). The under-representation of KIT exon 11 substituted GISTs in the advanced/metastatic GIST trials would suggest a more indolent clinical behavior. In a Norwegian population study, the presence of point mutations was associated with a low mitotic count [25]. In the Polish registry study tumors with KIT exon 11 substitutions were also characterized by low mitotic activity and average size $<5 \mathrm{~cm}$. Patients with $K I T$ substitutions have also been shown to have a better 5-year RFS rate (50.7\%) than those with KIT deletions or duplications (28.1 and $40.0 \%$, respectively) [14].

\section{Duplications in KIT exon 11}

Duplications in KIT exon 11 were historically associated with gastric tumor location and female gender, and also linked with a favorable clinical course [26]. The results of the Polish registry study confirmed this association since 26 out of 30 GISTs with $3^{\prime}$-end internal tandem duplications were of gastric origin and 21 of them were found in women. A relatively 
good clinical outcome has also been observed in patients with these tumors [14].

\section{Homo/hemizygous KIT exon 11 mutation status}

Loss of the WT KIT 11 allele and presence of homozygous KIT exon 11 mutations was strongly associated with a malignant clinical behavior in gastrointestinal stromal tumors [27]. In the Polish registry study $4 \%$ (17) of KIT exon 11 mutant GISTs lacked a WT allele [14], indicating a homo/hemizygous KIT status. Eleven of the 17 cases were associated with a high risk of metastatic disease or were already disseminated at the time of presentation, corroborating previous findings.

\section{Exon 9 KIT A502_Y503 duplication}

KIT mutations in exon 9 coding for the extracellular domain occur in $7-15 \%$ of GIST cases (Table 2). These mutations are believed to mimic the conformational change that the extracellular KIT receptor undergoes when ligand is bound. KIT exon 9 mutations characterized by A502-Y503 codon duplications are almost exclusively found in intestinal GISTs and for long they have been associated with a more aggressive phenotype [28].

In the Polish registry study KIT A502_ Y503dup mutation was not only associated with a small intestinal origin (27 out of 31 tumors) and malignant behavior, but also with a male predominance (21 out of 31) [14].

In contrast to previous observations Künstlinger and colleagues in 2013, described for the first time that nearly $20 \%$ of $K I T$ exon 9-mutated GISTs actually occur in the stomach or rectum. They provided evidence that exon 9-mutated GISTs metastasize significantly more often to the peritoneum than to the liver. Analyzing the data of over 1500 GISTs from their registry, KIT exon 9 mutations were neither associated with intermediate-risk/high-risk status nor overrepresented among metastatic lesions and thus they concluded that exon 9 mutations per se do not have a prognostic relevance [29].

In the ConticaGIST registry study when compared with KIT p.W557_K558del, KIT p.A502_Y503dup had a lower median mitotic rate, whereas there was no difference in tumor size for these mutants. Overall, both KIT exon 9 and KIT del-inc557/558 mutants were associated with relatively equal and inferior DFS (median DFS in both groups, 45.5 months; 5-year DFS,
37.9 and $33.1 \%$, respectively). There is solid evidence that compared with gastric tumors', small intestinal tumors with similar size and mitotic activity have a markedly worse prognosis. Across the board GISTs localized outside of the stomach were larger, had a higher mitotic rate and had worse 5-year DFS (34.2 vs $58.1 \%$ ) in comparison with gastric tumors. As mentioned above, comparison between tumors with KIT exon 9 and KIT exon 11 mutations (both, KIT del-inc557/558 and other KIT exon 11) of nongastric origin did not show differences in tumor clinical behavior as assessed by survival analysis. Thus, the authors concluded that in extra-gastric sites, the worse prognosis of KIT exon 9 mutants is related to the tumor location itself rather than to an intrinsic aggressive biologic nature of this mutation. In further support for this hypothesis, six out of seven gastric KIT exon 9 mutants in the ConticaGIST series study were classified as nonhigh risk, with only one of these patients developing progressive disease (PD), following a relatively long DFS of 56 months [13].

\section{Exon 13 \& 17}

It has been estimated that the frequency of exon 13 and 17 mutations is not higher than $1-2 \%$ [30]. In the Polish registry study KIT tyrosine kinase domain mutations involving exons 13 and 17 were found in two cases each, supporting the notion that these are uncommon mutations [14]. Mutations in exon 17 encoding the activation loop of the kinase seem to stabilize the active conformation. Primary mutations, such as that of K642E in exon 13 encoding the ATP-binding region are extremely rare and are speculated to interfere with the physiological auto-inhibitory function of the JM domain [30].

In a multicenter study of 54 cases with primary KIT exon 13 or exon 17 mutant GISTs, specifically among the KIT exon 17 mutants twice as many tumors arouse from the small bowel than the stomach. Intestinal site of origin of tumors was also over-represented among the KIT exon 13 mutants as compared with population-based studies. Overwhelming majority of the KIT exon 13 or exon 17 mutants displayed pure spindlecell morphology, very rarely associated with epithelioid features. Gastric KIT exon 13 mutant GISTs were slightly larger and of a higher risk group than gastric GISTs on average, whereas the behavior of small intestinal GISTs with KIT exon 13 or KIT exon 17 mutations did not differ from other small intestinal GISTs [30]. 


\section{- PDGFRA mutant GIST}

PDGFRA mutations are reported in $1.6-2.7 \%$ of GISTs in Phase III clinical trials enrolling patients with advanced disease and up to $12.9-14 \%$ of primary tumors in population studies (Table 2). The markedly lower representation of PDGFRA-mutated GISTs in clinical trial material can be easily explained by a comparatively benign clinical behavior of these tumors. Historically they were indeed correlated with a more indolent course of disease [31]. Moreover, PDGFRA mutant GISTs are almost exclusively (90-93\%) of prognostically more favorable gastric origin. The most prevalent genotype is the p.D842V substitution involving the second kinase domain (which corresponds to exon 17 of KIT), detected in $60-65 \%$ of all PDGFRA mutated tumors (Table 2). PDGFRA and KIT mutations are mutually exclusive and activate similar downstream signal transduction pathways [32].

In the Polish registry study, most changes among PDGFRA mutated tumors were identified in exon 18 (11.9\%), including the p.D842V substitution and with in-frame deletion or deletion/insertion of different lengths (9-15 bp) in 8.2 and $3.7 \%$ of the cases, respectively. Three cases $(0.7 \%)$ showed substitution in exon 14 of PDGFRA (p.N659K), and one (0.2\%) revealed deletion in exon 12 JM domain (p.S566_ E571delinsR). Multivariate analysis in this study revealed that patients with tumors with mutations involving PDGFRA, and KIT exon 11 substitutions or duplications have lower risk of 5-year relapse when compared with patients with KIT deletions involving codons 557/558 [14].

In the ConticaGIST series among tumors with PDGFRA mutations, the most prevalent was the p.D $842 \mathrm{~V}$ substitution $(9.8 \%$ of all mutations and $65.2 \%$ of the PDGFRA exon 18 mutations). PDGFRA exon 18 mutation status correlated with an extremely favorable disease outcome (median DFS not reached; 5-year DFS, $75 \%$ ) in comparison with other mutations. KIT exon 9 and KIT delinc557/558 mutations were associated with a significantly increased risk for tumor progression (HR: 1.47 and 1.45), in contrast with PDGFRA exon 18 mutation (HR: 0.23). There was no significant difference in DFS of PDGFRA p.D842V versus other PDGFRA exon 18 mutations. Notably, among gastric PDGFRA mutations, the vast majority that progressed (11 of 14) carried an exon 18 PDGFRA D842V substitution. While the number of the tumors with PDGFRA exon 18 mutations originating from nontypical anatomical sites was too low for a conclusive analysis, the frequency of relapse was lower in gastric versus nongastric PDGFRA exon 18 mutated tumors (11.8 vs $25 \%$ ), respectively [13].

It has been suggested that GISTs with the PDGFRA exon 14 mutation represent a subset of clinically favorable gastric tumors (exclusively gastric location) with almost exclusively epithelioid morphology [33].

\section{- KIT/PDGFRA mutation-negative 'WT' GIST}

Approximately $15 \%$ of adult GISTs do not have detectable mutations in KIT or PDGFRA and historically were simply referred to as 'WT' GISTs. KIT/PDGFRA WT GISTs express high levels of KIT and can arise from any part of the GI tract. Phosphorylated KIT is detectable in some of these tumors, suggesting KIT activation may still have a role in their pathophysiology [34].

\section{- SDH-deficient 'WT' GIST}

About half of all KIT/PDGFRA WT GISTs have inactivating mutations in the genes coding one of the four (SDHA, SDHB, SDHC and SDHD) subunits of the SDH complex. The SDH complex is located in the inner mitochondrial membrane and plays a role in the electron transport chain and the Szent-Györgyi-Krebs cycle by replacing succinate to fumarate. Either gene mutations in any member of the SDH complex or an as-yet-unknown mechanism destabilizes the SDH complex. SDH enzyme dysfunction leads to accumulation of succinate, resulting in HIF1- $\alpha$ stabilization and HIF1- $\alpha$ controlled oncogene transcription. In WT GISTs without SDH activity, upregulation of HIF1- $\alpha$ may lead to increased growth signaling through IGF1R and VEGFR [35].

Double hit inactivation of any components of the SDH complex destabilizes the entire complex, resulting in degradation of the SDHB subunit. Several international groups have demonstrated that all SDH mutations are reliably detected by SDHB loss on IHC, therefore, SDHB-IHC is currently the method of choice to test for SDH deficiency in KIT/PDGFRA WT GIST [35-37].

SDH gene germline mutations are features of the Carney-Stratakis syndrome, an inherited predisposition to multiple gastric GISTs and paragangliomas. Carney-triad is characterized by multiple gastric GISTs, paragangliomas and 
pulmonary chondromas with no SDH gene germline mutations detected (despite a deficiency of SDHB immunoreactivity) [38]. However, most recently it was suggested that in rare occasions the Carney triad can be allelic to CarneyStratakis syndrome. In the largest published cohort of 63 unrelated Carney triad patients six patients $(9.5 \%)$ were found to have germline variants in the $S D H A, S D H B$ or $S D H C$ genes [39]. Detecting SDH deficiency therefore, will trigger further clinical investigations in order to exclude syndromic GISTs, especially in younger patients.

SDH-deficient GISTs are particularly common in childhood and young adulthood, approximately $1-2 \%$ of all GISTs occurring in the pediatric population [40]. These SDH-deficient pediatric GISTs are characterized by unique clinical, morphological and genetic features. They have a predilection for the stomach, commonly demonstrate a multilobulated/multinodular growth pattern, frequently metastasize to lymph nodes and are mostly WT for KIT and PDGFRA [35,36].

Importantly SDH-deficient 'pediatric-type' GISTs also account for between 5 and $7.5 \%$ of all gastric GISTs occurring in adults [35]. It must be emphasized that the prognosis of SDH-deficient GISTs cannot be predicted by size and mitotic rate as even small, mitotically inactive SDHdeficient GISTs may metastasize. Interestingly when metastases do occur they may be strikingly indolent, sometimes remaining stable for years or decades [37]. As their distinctive histology predicts genotype and clinical behavior it can be recommended that IHC for SDHB should be performed on gastric GISTs with compatible 'pediatric-type' morphology.

In a recently published study $S D H A$ mutations were associated with statistically significant better clinical outcome as compared with KIT/PDGFRA mutations and KIT/PDGFRA WT without SDH deficiency. All survival analyses (from diagnosis of primary tumors and from diagnosis of metastatic disease) confirmed a far more indolent course of disease for patients with SDHA mutated WT GISTs [41].

\section{SDH proficient 'WT' GIST}

In the absence of the well-characterized frequent RTK mutations further, far less frequent mutations have been described in the members of the downstream signaling pathway, with mutations in BRAF (V600E), HRAS, NRAS or PIK3CA genes. These mutations presumably cause the constitutive activation of KIT downstream signal pathways. In addition, KIT/PDGFRA WT GIST may be related to syndromic neurofibromatosis type I (NF-1) disease, associated with NF1 protein loss of function due to genomic inactivation of the NF1 gene [5].

Hostein et al. screened 321 GISTs with 70 WT GISTs for $B R A F$ mutation. Similar to other tumor types where $B R A F$ mutations are more commonly observed, the mutations seen in GIST are also located within the exon 15 V600E hot spot. BRAF V600E was detected in nine $(13 \%)$ of the 70 WT GISTs and no mutations were detected in GISTs bearing KIT or PDGFRA mutations. Interestingly $B R A F$ $\mathrm{V} 600 \mathrm{E}$ detection in the tumor did not result in a higher expression of the B-raf protein or the preferential activation of the p42/44 MAPK signaling pathway compared with GISTs without the BRAF mutation [42]. KIT, PDGFRA and $B R A F$ WT GISTs are commonly referred to as 'triple-negative' GISTs.

Miettinen and colleagues noted that patients with NF1 are overrepresented by at least 45-fold among GIST patients and GISTs occur in approximately $5-25 \%$ of NF1 patients. NF1-associated GISTs develop secondary to a somatic inactivation of the WT NF1 allele in the tumor and are commonly multicentric, predominantly located in the small intestine and lack KIT and PDGFRA mutations. The majority of NF1-associated GISTs present as small, low mitotic index lesions and they are associated with quite favorable long-term clinical outcomes reflected in low recurrence and metastases rates. Interestingly NF-1 associated GISTs arising from the duodenum display an aggressive behavior, being large mitotically active tumors with pronounced metastatic potential [43].

A small subgroup of KIT/PDGFRA WT GIST, referred as 'quadruple WT GIST' (Q-WT GIST), that lack mutations in any of the known KIT exons $(8,9,11,13,14,17)$ or PDGFRA exons $(12,14,18)$ or RAS pathways, including $B R A F$ (exons 11, 15) and $R A S$ (exons 2, 3), or $N F 1$, and yet retain an intact SDH complex (SDHB IHC positive, and no mutations in $S D H$ ) has been identified. The true clinical relevance of this subgroup is yet to be elucidated [44].

Most recently two oncogenic RTK translocations were reported in a subset of nongastric Q-WT GISTS [45,46]. Two cases with ETV6NTRK3 fusion (in a colonic and a rectal Q-WT GIST) and one with FGFR1-TACC1 
translocation (in a small intestinal Q-WT GIST) were found. Both of these alterations have been identified in other malignancies and are known to constitutively activate the target kinases. These RTK translocations found in a subset of Q-WT GIST seem to define a novel biological class of GIST arising in nongastric sites. The oncogenic RTK fusion proteins could represent potential therapeutic targets. If confirmed in larger series, routine testing for RTK translocation may be indicated for Q-WT GIST [45,46].

\section{- Genetically unstable GIST}

The accumulation of chromosomal abnormalities correlates with the biological behavior of GISTs. About two-thirds of GISTs exhibit 14 monosomy or partial loss of $14 \mathrm{q}$, and half of them have loss of the long arm of chromosome 22. Chromosome 14 or 22 aberrations are linked to a borderline malignant potential. An aggressive biology is associated with the loss of chromosome 1p, 9p (spanning CDKN2A or p16INK4A) and 11p regions. Gain in chromosomal segments $8 \mathrm{q}$ and $17 \mathrm{q}$ have been associated with increased metastatic potential. It is an interesting observation that while an unstable karyotype correlates with the presence of GIST mutations, KIT/PDGFRA WT GISTs are genomically stable $[47,48]$.

Combined prognostic value of mutational status in treatment-naive GIST

As detailed above individual genetic alterations, for instance KIT exon 11 deletions, were reported already a decade ago as an independent adverse prognostic factor in patients with untreated GIST [11]. However, until very recently, the combined prognostic value of the most relevant genetic GIST subtypes has not been elucidated. Rossi et al. analyzed a series of 451 untreated primary localized GISTs for KIT, PDGFRA and BRAF mutations [49] and found that mutational status is a significant prognostic indicator of overall survival (OS) in treatment-naive, localized GISTs. Patients with $K I T$-mutated tumors had a worse outcome than PDGFRA-mutated or 'triple-negative' (KIT, PDGFRA, BRAF WT) cases. Based on multivariable Cox regression models the authors identified three distinct molecular risk groups. Group I, consisting of PDGFRA exon 12, BRAF and $K I T$ exon 13-mutated cases, exhibited the best clinical outcome. The intermediate risk (HR: 3.06) Group II, included 'triple-negative',
KIT exon 17, PDGFRA exon $18 \mathrm{D} 842 \mathrm{~V}$ and PDGFRA exon 14-mutated GISTs. Group III, comprised of KIT exon 9 and exon 11 and PDGFRA non-D842V exon 18 mutant GISTs, displayed the worst clinical outcome (HR: 4.52). This study clearly highlighted the prognostic impact of mutational status on the natural history of GIST. Inclusion of molecular prognostic grouping into currently used clinicopathologic risk stratification criteria could clearly fine tune the decision-making process for adjuvant therapy.

\section{- Therapeutic implications of different genetic subtypes of GIST}

Due to its ubiquitous role in the pathogenesis of GISTs, KIT has become a universal therapeutic target. Imatinib, a competitive inhibitor of the ATP-binding domain can only bind to the inactive conformation of KIT and its pronounced clinical efficacy was confirmed one and a half decades ago [50,51]. Imatinib represents the standard upfront medication for the treatment of advanced/metastatic GIST, with an overall $80 \%$ disease control rate (objective response or stable disease), a median progression-free survival (PFS) of approximately 20 months and a median OS of around 50 months [52,53].

Over the last decade KIT mutational status has emerged as a strong predictive indicator of treatment response. GISTs with KIT exon 11 mutated genotype show a marked, approximately $70 \%$ objective response rate (ORR; complete response/partial response) as compared with the 40-45\% ORR seen in exon 9 mutant and WT GISTs. The ORR advantage seen in exon 11 mutant patients translates into an additional OS gain of approximately 20 months for this subgroup compared with exon 9 mutated and 'WT' GISTs (60 vs 40 months for exon 9 mutant and WT GIST, respectively) [52,53].

In the pooled analysis of the two pivotal Phase III trials comparing 400 versus $800 \mathrm{mg}$ daily imatinib dose, the sole predictive factor of response was the presence of a KIT exon 9 mutation. The estimated risk of progression or death was reduced by $42 \%$ in the high-dose arm (compared with the standard-dose arm) in patients with KIT exon 9 mutated tumors. However, no significant difference in OS was seen between patients treated with 400 and $800 \mathrm{mg}$ imatinib, irrespective of mutational status. The study concluded that for most patients, the recommended daily dose is $400 \mathrm{mg}$ daily, with the exception of 
KIT exon 9 mutated tumors where the $800 \mathrm{mg}$ dose can be considered [54].

Approximately, 5\% of patients with GIST do not express KIT, but may have tumors that harbor imatinib-sensitive KIT or PDGFRA mutations; therefore, patients with KIT-negative GISTs should not, a priori, be denied imatinib therapy and tumor samples should be sent for mutational analysis [55].

Despite majority of GIST patients benefiting from imatinib treatment, approximately $10-15 \%$ of them show primary resistance with early progression within 3-6 months of initiating therapy, in keeping with primary resistance. Although $80-85 \%$ patients with advanced GIST benefit from imatinib treatment, 40-50\% of patients subsequently develop secondary resistance to the agent with a median time to progression of about 24 months $[20,52,56]$.

\section{- Primary imatinib resistance}

The accumulated results of preclinical and clinical research have provided powerful tools in the explanation, prediction and management of primary resistance. Both primary and secondary resistance to imatinib can be partially explained by a conformational shift in the kinase domain of KIT and PGFRA that favors the activated state [51]. Preclinical data indicated that PDGFRA isoforms with a substitution involving codon $D 842$ in exon 18 (D842V, RD841-842KI, DI842-843IM) lead to primary resistance to imatinib, with the exception of D842Y. Other mutations in exon 18 (D846Y, N848K, Y849K and HDSN845-848P) were found to be imatinib sensitive. Imatinib can only bind to the inactive conformation of both the KIT and PDGFRA receptors. PDGFRA D842V mutation results in a distortion of the kinase activation loop, thus strongly tilting the protein conformation in favor of the activated structure. In clinical trials PDGFRA mutant GISTs showed a mild sensitivity to drug (66\%) except the exon $18 \mathrm{D} 842 \mathrm{~V}$ mutation which proved to be resistant [15,57]. However, a most recent large multicenter observational study reported objective response to imatinib in a small proportion of patients with PDGFRA D842V-mutated GIST. Out of 16 patients with the mutation $12.5 \%$ had partial response, $18.8 \%$ had stable disease and $56.3 \%$ had PD as best response according to Choi criteria. Median time to progression was 8.0 months (range: $0-42$ ). The authors of the report rightly concluded that as patients with
PDGFRA D842V-mutated GIST do have a small chance of responding to standard TKI treatment, imatinib should not be universally denied in patients harboring this mutation [58].

It is currently unclear how the predictive power of genetic alterations seen in the advanced setting may translate into decision-making regarding adjuvant therapy. The two adjuvant trials (the USA ACOSOG Z 9001 and the German-Scandinavian study) have failed to demonstrate that a specific mutation can predict a better RFS and OS in treated patients [16,59].

In the ACOSOG Z 9001 trial on multivariable analysis of patients tumor genotype was not significantly associated with RFS, however patients with KIT exon 11 deleted tumors assigned to 1 year of adjuvant imatinib did have a longer RFS. The excellent survival of patients with tumors harboring PDGFRA mutations in the placebo group provides a good argument that these patients may not require adjuvant treatment [16]. In the German-Scandinavian study KIT exon 11 mutant GIST patients benefited from the longer 3-year treatment, whereas no significant improvement over 12 months of imatinib was found in KIT exon 9 or PDGFRA mutant or WT patients (note that the number of patients were small in these later categories) [59].

In a small series of seven metastatic NF1associated GIST patients, three of the four imatinib-treated patients showed primary resistance to the treatment (all three tumors were 'WT' GISTs). The fourth metastatic patient with an exon 18 mutated tumor had temporary stable disease. Median OS for this four-patient cohort was 21 months [60].

Rege and colleagues in their seven patient cohort of metastatic 'pediatric-type' SDHdeficient adult GISTs reported absolute primary resistance to imatinib in all of the cases. In contrast to this, in a more recent 2015 report 4/5 of imatinib-treated 'pediatric-type' SDHAmutant metastatic GISTs showed a longer than 6-month PFS, with two ongoing responses at 19 and 58 months [61]. This later observation begs the question whether SDHA mutational status shall be performed routinely in SDH-deficient tumors as assessed by IHC.

Alternate signaling pathway mutations (like $B R A F$ exon 15 activating mutation) in patients lacking identifiable PDGFRA or KIT mutations can be potential alternative mechanisms explaining their frequent primary resistance to imatinib [62-64]. 
- Secondary imatinib resistance

Acquisition of KIT or PDGFRA secondary mutations represent the most frequent mechanism of imatinib resistance in GIST [64,65]. Radiological evidence of clonal resistance can be detected as the appearance of one or more areas of increased vascularity within a previously responding or stable lesion. These lesions can precede by several months PD) according to Response Evaluation Criteria In Solid Tumors (RECIST). In a study of TKI pretreated patients [66], the mutational status of individual nodules within a dominant tumor mass confirmed intra- and inter-lesional mutational heterogeneity between co-existing nodules. In total, $83 \%$ of samples had at least one secondary mutation, $67 \%$ had two to five different secondary mutations in different tumor samples and $34 \%$ had more than two mutations in the same clone. Secondary mutations clustered in the KIT ATP-binding pocket (exon 13) and kinase activation loop (exon 17). KIT amplification was detected by FISH in two of the ten metastases lacking secondary KIT mutations. No KIT kinase resistance mutations were detected in KIT/PDGFRA WT GISTs or in $K I T$-mutant GISTs showing unusual morphology and/or loss of KIT expression by IHC. These results clearly expose the emergence of heterogeneous resistance mechanisms in these tumors. Furthermore, these data indicate that repeat biopsy (with mutational analysis) may not be relevant in metastatic GIST and highlight the therapeutic challenges involved in managing heavily pretreated patients [66]. Emergence of polyclonal imatinib resistance poses a great difficulty in developing effective single agent next-generation kinase inhibitors.

Secondary KIT exon 17 mutations affecting the activation loop stabilize the active receptor conformation, while imatinib can only bind and inhibit the nonactivated (auto-inhibited) conformation of KIT. Activation loop mutations therefore indirectly induce imatinib resistance by shifting the equilibrium strongly in favor of the active conformation [21].

In a Phase I/II trial half of the patients progressing on imatinib had a secondary KIT mutation [67]. Mutational distribution was nonrandom, secondary mutations clustering in exons 13 and 14, encoding the drug/ATP-binding pocket of the receptor and in exon 17, encoding the kinase activation loop. The most commonly detected secondary mutation was the exon 13 V654A. Two tumors had secondary mutations in
KIT exon 18. One patient had distinct secondary mutations in separate lesions, that of exon 13 V654A and exon $17 \mathrm{D} 816 \mathrm{H}$ respectively. Of the four primary PDGFRA mutant samples one had a secondary mutation in exon 18 (primary mutation in exon 12), two lacked secondary mutations (both had primary exon $18 \mathrm{D} 842 \mathrm{~V}$ mutations) and in the fourth case there was no postimatinib sample available. More interestingly, in the post-imatinib samples of eight KIT or PDGFRA wild type GISTs no secondary mutations were found. Secondary mutations were more likely to be found in patients who initially harbored KIT exon 11 mutations (73\%) as compared with KIT exon 9 mutations (19\%). The higher rate of secondary mutations in the primary exon 11 mutant subgroup, is likely to be due to the longer exposure to imatinib. In preclinical in vitro studies sunitinib potently inhibited the kinase activity of KIT receptors that contained imatinib-resistant secondary mutations in the drug/ATP-binding pocket, such as V654A (exon 13) and T670I (exon 14). Furthermore, in vitro sunitinib was relatively ineffective at inhibiting KIT receptors with secondary mutations affecting the activation loop. In keeping with these findings, clinical trial data have shown that PFS and OS were longer and the clinical benefit rate was higher for patients with tumors harboring the KIT exon 13 or 14 ATP-binding-pocket mutations than those with $K I T$ exon 17 or 18 activation loop mutations [67].

Amplification of the KIT and/or PDGFRA genes has also been postulated as a potential mechanism for either primary or delayed TKI resistance $[67,68]$. Some GISTs lacking secondary kinase mutations do show genomic amplification of KIT and/or become hemi- and/or homo-zygous for the primary KIT mutation undergoing deletion of the WT KIT allele [68].

$I G F 1 R$ amplification may represent another mechanism of de novo or acquired imatinib resistance. It has to be noted that IGF1R overexpression was detected in the overwhelming majority $(89 \%)$ of SDH-negative gastric GISTs but in only $1 \%$ of SDHB-positive gastric GISTs [69].

\section{- Treatment beyond imatinib}

Sunitinib and regorafenib have been approved for imatinib-resistant GIST based on placebo controlled randomized Phase III trials [70,71].

The response to second-line sunitinib correlates with the primary (pre-imatinib) tumor 
mutation status [67,72]. In a worldwide, openlabel treatment-use study of 1124 patients resistant or intolerant to imatinib a median PFS of 7.1 months was reported on sunitinib. The PFS in patients with a primary KIT exon 9 mutation was significantly better as compared with those with primary exon 11 mutation, with figures of 12.3 and 7.0 months, respectively. Moreover, primary KIT exon 9 mutation was associated with a longer OS and higher ORR as compared with exon 11 GISTs [72].

As far as secondary KIT mutations are concerned, for primarily KIT mutant GIST patients the median PFS and OS with sunitinib was significantly longer for secondary KIT exon 13 or 14 mutations than for those with secondary exon 17 or 18 mutations [67]. These results corroborate the in vitro findings which showed that sunitinib effectively inhibits the phosphorylation of KIT double mutants where secondary mutation affected the drug/ATP-binding pocket. On the other hand, sunitinib has very little activity against KIT double mutants where secondary mutations affected the activation loop [73].

Moreover, preclinical studies of imatinibresistant GIST cell lines evaluating sunitinib sensitivity showed that only KIT T670I (gatekeeper) and V654A mutants were sensitive to sunitinib, while PDGFRA D842V (activation loop) mutants remained resistant [73]. Unfortunately, the available clinical data are yet too limited to investigate the effects of PDGFRA mutations on efficacy outcomes following sunitinib treatment [67,72].

There is little evidence available in regard of the predictive power of mutational status in the third-line regorafenib treatment of GISTs; however, regorafenib showed efficacy in all genetic subtypes of GISTs including exon 9 and PDGFRA D842V mutants [71,74].

\section{Conclusion}

The discovery of activating mutations in $K I T$ and PDGFRA and their role in the pathogenesis of GIST has revolutionized our understanding of the biology and therapy of this disease. The introduction of imatinib has ultimately led to a radical improvement in life expectancy of advanced GIST patients. However, it is clear that GISTs are composed of many different molecular subtypes, with differing clinical characteristics and response to therapy. The detection of specific somatic genetic changes in GISTs can provide potential predictive and prognostic information for guiding therapy. We can conclude that the term 'WT' GIST is outdated by a series of recent findings, and in case it is used reference always should be made which genes it applies to (i.e., KIT/PDGFRA WT GIST or KITIPDGFRA/BRAFWT GIST and so on).

Nonhot spot exon 8 and exon 14 mutations of the KIT receptor are extremely rare and are not routinely screened for. There is a genuine concern that without identifying these rare mutations we miss to identify vital information about the biological behavior and treatment sensitivity (both in the adjuvant and metastatic setting) of these genetic subtypes. KIT exon 11 mutant patients are twice as likely to respond to imatinib than those with exon 9 mutant or WT GISTs. Moreover, higher response rates to imatinib in the KIT exon 11 mutant group translates into a PFS and OS advantage as compared with all non-KIT exon 11 mutant GISTs. The presence of a $K I T$ del-inc557/558 mutation in a study of patients from the pre-imatinib era, reported that this mutation was strongly associated with poorer outcomes both in high-risk and nonhigh-risk gastric GIST, indicating its additional prognostic value for patient selection for adjuvant therapy. It remains to be shown whether patients with GISTs with KIT del-inc557/558 genotype should not be kept longer (or even lifelong) on standard adjuvant imatinib treatment (i.e., 3 years).

KIT exon 9 mutations have been associated with a poor clinical prognosis as compared with other mutations. However, the worse prognosis of KIT exon 9 mutants seems to be related to the almost exclusive high-risk extra-gastric tumor location of these GISTs rather than to an intrinsic aggressive biologic behavior. KIT exon 9 mutant GISTs are more likely to respond to the higher $800 \mathrm{mg}$ dose of imatinib, and in the advanced setting patients there is a strong recommendation of starting patients with tumors harboring theses mutations on the higher dose. The optimal dose of imatinib in the adjuvant setting for KIT exon 9 mutant GISTs is yet to be elucidated. Identification of PDGFRA mutations in GIST proved to be a strong predictor of good clinical outcome and this molecular factor could therefore add a significant value to the current consensus risk criteria used for GIST stratification. In addition, given that these mutations comprise the majority of cases with the imatinibresistant p.D842V subtype, mutational testing is highly relevant in order to avoid overtreatment 
of gastric tumors with imatinib in the adjuvant setting. As tumors with certain mutations such as PDGFRA D842V mostly have primary resistance to imatinib and sunitinib, patients should be offered participation in clinical trials of novel agents. A recent large multicenter observational study has suggested that some tumors with the D842V mutation may respond to standard tyrosine kinase therapy. While tumors with no PDGFRA or KIT mutations (WT GISTs) are generally considered to be less responsive to these agents, more focus should be directed at the exact mutational landscape of these cancers with regard to the therapeutic decision-making process. These are important observations that should be factored into the decision-making process prior to commencing tyrosine kinase therapy.

\section{Future perspective}

Inevitably all currently available standard treatment options lose their efficacy to control advanced GIST. Ongoing preclinical and clinical research is focusing on evaluating novel therapeutic approaches to overcome primary and secondary resistance to imatinib and the other two currently available licensed medications, sunitinib and regorafenib. Targeting deregulated downstream pathways shall provide further treatment options in the management of imatinib/sunitinib/regorafenib insensitive/resistant GISTs.

Emerging information about the prognostic value of different, specific mutations warrants further evaluation, possibly using pooled cohorts stratified for the mutational status from the available prospective clinical trials in the adjuvant setting.

Currently, adjuvant therapy in 'high-risk' WT GIST and the optimal systemic treatment for metastatic WT GIST remain debatable 'hot topic' questions, with no clear-cut guidelines. The group of WT patients is heterogeneous and further attempts should be made to further delineate individual molecular subtypes, in order to guide future clinical trial development and optimize systemic therapy.

\section{EXECUTIVE SUMMARY}

\section{Classification of gastrointestinal stromal tumors}

- Gastrointestinal stromal tumors (GISTs) are the most common type of mesenchymal tumors of the digestive tract, mainly defined by the presence or lack of mutually exclusive gain-of-function mutations in the KIT and PDGFRA receptors.

- GISTs historically referred to as 'wild-type' (WT) GISTs are a very heterogeneous group which do not have a detectable mutation in either the KIT or the PDGFRA receptor genes, while KIT activation by phosphorylation is still detectable in these tumors.

- WT GISTs can be split into two large groups, based on whether they are proficient or deficient in the succinate dehydrogenase complex.

- Detecting mutations in alternative pathways can further subclassify WT GISTs.

\section{Therapeutic implications of genetic subtypes}

- KIT exon 11 mutant patients are twice as likely to respond to imatinib than those with exon 9 mutant or WT GISTs with a progression-free and overall survival advantage as compared with all non-KIT exon 11 mutant GISTs.

- KIT exon 9 mutant GISTs are more likely to respond to the higher $800 \mathrm{mg}$ dose of imatinib, and in the advanced setting patients can be started $a b$ ovo on the higher dose.

- The PDGFRA D842V isoform with a substitution involving codon D842 in exon 18 is generally believed to lead to primary imatinib resistance. Caution should be exercised when it comes to therapeutic decisions as recent data suggest some response to imatinib in this subset of tumors.

- While SDH-deficient 'pediatric-type' GISTs have been previously attributed absolute primary imatinib resistance, most recent reports suggest imatinib responsiveness in SDHA-mutated tumors.

- Acquisition of secondary mutations in either KIT or PDGFRA represents the most frequent mechanism of imatinib resistance in GIST.

- Adjuvant therapy in 'high-risk' WT GIST and the optimal systemic treatment for metastatic WT GIST remain debatable 'hot topic' questions, with no clear-cut clinical guidelines. 


\section{Financial \& competing interests disclosure}

The authors have no relevant affiliations or financial involvement with any organization or entity with a financial interest in or financial conflict with the subject matter or materials discussed in the manuscript. This includes employment, consultancies, honoraria, stock ownership or options, expert testimony, grants or patents received or pending, or royalties.
No writing assistance was utilized in the production of this manuscript.

\section{Open access}

This work is licensed under the Creative Commons Attribution-NonCommercial 4.0 Unported License. To view a copy of this license, visit http://creativecommons.org/ licenses/by-nc-nd/4.0/

\section{References}

Papers of special note have been highlighted as:

- of interest; $\bullet \bullet$ of considerable interest

1 Miettinen M, Lasota J. Gastrointestinal stromal tumours: pathology and prognosis at different sites. Semin. Diagn. Pathol. 23, 70-83 (2006).

2 Huizinga JD, Thuneberg L, Klüppel M et al. $\mathrm{W} /$ kit gene required for interstitial cells of Cajal and for intestinal pacemaker activity. Nature 373(6512), 347-349 (1995).

3 DeMatteo RP, Lewis JJ, Leung D et al. Two hundred gastrointestinal stromal tumours: recurrence patterns and prognostic factors for survival. Ann. Surg. 231, 51-58 (2000).

4 Rossi S, Miceli R, Messerini L et al. Natural history of imatinib-naive GISTs: a retrospective analysis of 929 cases with long-term follow-up and development of a survival nomogram based on mitotic index and size as continuous variables. Am. J. Surg. Pathol. 35, 1646-1656 (2011).

5 Yamamoto H, Oda Y. Gastrointestinal stromal tumour: recent advances in pathology and genetics. Pathol. Int. 65(1), 9-18 (2015).

6 Nocka K, Majumder S, Chabot B et al. Expression of c-kit gene products in known cellular targets of W mutations in normal and W mutant mice-evidence for an impaired c-kit kinase in mutant mice. Genes Dev. 3(6), 816-826 (1989).

7 Besmer P, Murphy JE, George PC et al. A new acute transforming feline retrovirus and relationship of its oncogene $\mathrm{v}$-kit with the protein kinase gene family. Nature 320(6061), 415-421 (1986).

8 Hanks SK, Quinn AM, Hunter T. The protein kinase family: conserved features and deduced phylogeny of the catalytic domains. Science 241, 42-52 (1988).

9 Miettinen M, Sobin LH, Sarlomo-Rikala M. Immunohistochemical spectrum of GISTs at different sites and their differential diagnosis with a reference to CD117 (KIT). Mod. Pathol. 13(10), 1134-1142 (2000).

10 Lennartsson J, Jelacic T, Linnekin D et al. Normal and oncogenic forms of the receptor tyrosine kinase kit. Stem Cells 23(1), 16-43 (2005).

11 Huss S, Künstlinger H, Wardelmann E, Kleine MA et al. A subset of gastrointestinal stromal tumours previously regarded as wild-type tumours carries somatic activating mutations in KIT exon 8 (p.D419del). Mod. Pathol. 26(7), 1004-1012 (2013).

12 Debiec-Rychter M, Sciot R, Le Cesne A et al. EORTC Soft Tissue and Bone Sarcoma Group; Italian Sarcoma Group; Australasian GastroIntestinal Trials Group. KIT mutations and dose selection for imatinib in patients with advanced gastrointestinal stromal tumours. Eur. J. Cancer 42 (8), 1093-1103 (2006).

13 Wozniak A, Rutkowski P, Schöffski P et al. Tumour genotype is an independent prognostic factor in primary gastrointestinal stromal tumours of gastric origin: a European multicenter analysis based on ConticaGIST. Clin. Cancer Res. 20 (23), 6105-6116 (2014).

-. Provides invaluable information on the prognostic value of different genetic subtypes of gastrointestinal stromal tumor (GIST).

14 Wozniak A, Rutkowski P, Piskorz A et al. Polish Clinical GIST Registry. Prognostic value of KIT/PDGFRA mutations in gastrointestinal stromal tumours (GIST): Polish Clinical GIST Registry experience. Ann. Oncol. 23(2), 353-360 (2012).

-• Provides important information on the prognostic value of different genetic subtypes of GIST.

15 Heinrich MC, Owzar K, Corless CL et al. Correlation of kinase genotype and clinical outcome in the North American Intergroup Phase III Trial of imatinib mesylate for treatment of advanced gastrointestinal stromal tumour: CALGB 150105 Study by Cancer and Leukemia Group B and Southwest Oncology Group. J. Clin. Oncol. 26(33), 5360-5367 (2008).

- Landmark Phase III clinical trial providing important information on the predictive value of different genetic subtypes of GIST.
16 Corless CL, Ballman KV et al. Pathologic and molecular features correlate with long-term outcome after adjuvant therapy of resected primary GI stromal tumour: the ACOSOG Z9001 trial. J. Clin. Oncol. 232(15), 1563-1570 (2014).

17 Heinrich MC, Rubin BP, Longley BJ et al. Biology and genetic aspects of gastrointestinal stromal tumours: KIT activation and cytogenetic alterations. Hum. Pathol. 33(5), 484-495 (2002).

18 Hirota S, Isozaki K, Moriyama Y et al. Gain-of-function mutations of c-kit in human gastrointestinal stromal tumours. Science 279(5350), 577-580 (1998).

19 Chen LL, Holden JA, Choi $\mathrm{H}$ et al. Evolution from heterozygous to homozygous KIT mutation in gastro-intestinal stromal tumour correlates with the mechanism of mitotic nondisjunction and significant tumour progression. Mod. Pathol. 21, 826-836 (2008).

20 Sciot R, Debiec-Rychter M, Daugaard S et al. EORTC Soft Tissue and Bone Sarcoma Group; Italian Sarcoma Group; Australasian Trials Group. Distribution and prognostic value of histopathologic data and immunohistochemical markers in gastrointestinal stromal tumours (GISTs): an analysis of the EORTC Phase III trial of treatment of metastatic GISTs with imatinib mesylate. Eur. J. Cancer 44(13), 1855-1860 (2008).

21 Gajiwala KS, Wu JC, Christensen J et al. KIT kinase mutants show unique mechanisms of drug resistance to imatinib and sunitinib in gastrointestinal stromal tumour patients. Proc. Natl Acad. Sci. USA 106(5), 1542-1547 (2009).

22 Wardelmann E, Losen I, Hans V et al. Deletion of Trp-557 and Lys-558 in the juxtamembrane domain of the c-kit protooncogene is associated with metastatic behaviour of gastrointestinal stromal tumours. Int. J. Cancer 106, 887-895 (2003).

23 Martín J, Poveda A, Llombart-Bosch A et al. Deletions affecting codons 557-558 of the c-KIT gene indicate a poor prognosis in 
patients with completely resected gastrointestinal stromal tumours: a study by the Spanish Group for Sarcoma Research (GEIS). J. Clin. Oncol. 23, 6190-6198 (2005).

24 Martin-Broto J, Gutierrez A, Garcia-delMuro X et al. Prognostic time dependence of deletions affecting codons 557 and/or 558 of KIT gene for relapse-free survival (RFS) in localized GIST: a Spanish Group for Sarcoma Research (GEIS) Study. Ann. Oncol. 21, 1552-1557 (2010).

25 Steigen SE, Eide TJ, Wasag B et al. Mutations in gastrointestinal stromal tumours - a population-based study from northern Norway. APMIS 115, 289-298 (2007).

26 Lasota J, Dansonka-Mieszkowska A, Stachura $\mathrm{T}$ et al. Gastrointestinal stromal tumours with internal tandem duplications in 3 ' end of $K I T$ juxtamembrane domain occur predominantly in stomach and generally seem to have a favorable course. Mod. Pathol. 16, 1257-1264 (2003).

27 Lasota J, vel Dobosz AJ, Wasag B et al. Presence of homozygous KIT exon 11 mutations is strongly associated with malignant clinical behaviour in gastrointestinal stromal tumours. Lab Invest. 87, 1029-1041 (2007).

28 Antonescu CR, Sommer G, Sarran L et al. Association of KIT exon 9 mutations with nongastric primary site and aggressive behaviour: KIT mutation analysis and clinical correlates of 120 gastrointestinal stromal tumours. Clin. Cancer Res. 9, 3329-3337 (2003).

29 Künstlinger $\mathrm{H}$, Huss $S$, Merkelbach-Bruse $S$ et al. Gastrointestinal stromal tumours with KIT exon 9 mutations: update on genotype-phenotype correlation and validation of a high-resolution meltingassay for mutational testing. Am. J. Surg. Pathol. 37(11) 1648-1659 (2013).

30 Lasota J, Corless CL, Heinrich MC et al. Clinico-pathologic profile of gastrointestinal stromal tumours (GISTs) with primary KIT exon 13 or exon 17 mutations: a multicenter study on 54 cases. Mod. Pathol. 21, 476-484 (2008).

31 Lasota J, Dansonka-Mieszkowska A et al. A great majority of GISTs with PDGFRA mutations represent gastric tumours of low or no malignant potential. Lab Invest. 84, 874-883 (2004).

32 Lasota J, Miettinen M. KIT and PDGFRA mutations in gastrointestinal stromal tumours (GISTs). Semin. Diagn. Pathol. 23, 91-102 (2006).

33 Lasota J, Stachura J, Miettinen M. GISTs with PDGFRA exon 14 mutations represent subset of clinically favorable gastric tumours with epithelioid morphology. Lab. Invest. 86(1) 94-100 (2006).

34 Janeway KA, Liegl B, Harlow A et al. Pediatric KIT wild-type and platelet-derived growth factor receptor alpha-wild-type gastrointestinal stromal tumours share KIT activation but not mechanisms of genetic progression with adult gastrointestinal stromal tumours. Cancer Res. 67(19), 9084-9088 (2007).

35 Gill AJ. Succinate dehydrogenase (SDH) and mitochondrial driven neoplasia. Pathology 44, 285-292 (2012).

36 Miettinen M, Wang ZF, Sarlomo-Rikala M et al. Succinate dehydrogenase-deficient GISTs: A clinicopathologic, immunohistochemical, and molecular genetic study of 66 gastric GISTs with predilection to young age. Am. J. Surg. Pathol. 35, 1712-1721 (2011).

37 Miettinen M, Killian JK, Wang ZF et al. Immunohistochemical loss of succinate dehydrogenase subunit A (SDHA) in gastrointestinal stromal tumours (GISTs) signals SDHA germline mutation. Am. J. Surg. Pathol. 37(2), 234-240 (2013).

38 Stratakis CA, Carney JA. The triad of paragangliomas, gastric stromal tumours and pulmonary chondromas (Carney triad), and the dyad of paragangliomas and gastric stromal sarcomas (Carney-Stratakis syndrome): molecular genetics and clinical implications. J. Intern. Med. 266, 43-52 (2009).

39 Boikos SA, Xekouki P, Fumagalli E et al. Carney triad can be (rarely) associated with germline succinate dehydrogenase defects. Eur. J. Hum. Genet. 24(4) 569-573 (2016).

40 Janeway KA, Weldon CB. Pediatric gastrointestinal stromal tumour. Semin Pediatr. Surg. 21(1), 31-43 (2012).

41 Pantaleo MA, Lolli C, Nannini M et al. Good survival outcome of metastatic SDH-deficient gastrointestinal stromal tumours harbouring SDHA mutations. Genet. Med. 17(5), 391-395 (2015).

42 Hostein I, Faur N, Primois C et al. BRAF mutation status in gastrointestinal stromal tumours. Am. J. Clin. Pathol. 133(1), 141-148 (2010).

43 Miettinen M, Fetsch JF, Sobin LH et al. Gastrointestinal stromal tumours in patients with neurofibromatosis 1: a clinicopathologic and molecular genetic study of 45 cases. Am. J. Surg. Pathol. 30(1), 90-96 (2006).

44 Pantaleo MA, Nannini M, Corless CL et al. Quadruple wild-type (WT) GIST: defining the subset of gist that lacks abnormalities of
KIT PDGFRA SDH or RAS signaling pathways. Cancer Med. 4, 101-103 (2015).

45 Brenca M, Rossi S, Polano M et al. Transcriptome sequencing identifies ETV6-NTRK3 as a gene fusion involved in GIST. J. Pathol. 238(4), 543-549 (2016).

46 Heinrich M, Kang G, Warrick A et al. Oncogenic receptor tyrosine kinase (RTK) translocations in a subset of quadruple wild-type gastrointestinal stromal tumours (GIST). J. Clin. Oncol. 34(Suppl), Abstract 11012 (2016).

47 Assämäki R, Sarlomo-Rikala M, LopezGuerrero et al. Array comparative genomic hybridization analysis of chromosomal imbalances and their target genes in gastrointestinal stromal tumours. Genes Chromosomes Cancer 46, 564-576 (2007).

48 Wozniak A, Sciot R, Guillou L et al. Array $\mathrm{CGH}$ analysis in primary gastrointestinal stromal tumours: Cytogenetic profile correlates with anatomic site and tumour aggressiveness, irrespective of mutational status. Genes Chromosomes Cancer 46, 261-276 (2007).

49 Rossi S, Gasparotto D, Miceli R et al. KIT PDGFRA and BRAF mutational spectrum impacts on the natural history of imatinibnaive localized GIST: a population-based study. Am. J. Surg. Pathol. 39 (7), 922-930 (2015).

50 Joensuu H, Roberts PJ, Sarlomo-Rikala M et al. Effect of the tyrosine kinase inhibitor STI571 in a patient with a metastatic gastrointestinal stromal tumour. N. Engl. J. Med. 344(14), 1052-1056 (2001).

51 Roskoski R Jr. Structure and regulation of Kit protein-tyrosine kinase-the stem cell factor receptor. Biochem. Biophys. Res. Commun. 338(3), 1307-1315 (2005).

52 Blanke CD, Rankin C, Demetri GD et al. Phase III randomized, intergroup trial assessing imatinib mesylate at two dose levels in patients with unresectable or metastatic gastrointestinal stromal tumours expressing the kit receptor tyrosine kinase: $\mathrm{S} 0033$. J. Clin. Oncol. 26(4), 626-632 (2008).

53 Verweij J, Casali PG, Zalcberg J et al. Progression-free survival in gastrointestinal stromal tumours with high-dose imatinib: randomised trial. Lancet 364(9440), 1127-1134 (2004).

54 Gastrointestinal Stromal Tumour MetaAnalysis Group (MetaGIST). Comparison of two doses of imatinib for the treatment of unresectable or metastatic gastrointestinal stromal tumours: a meta-analysis of 1,640 patients. J. Clin. Oncol. 28(7), 1247-1253 (2010). 
- Important meta-analysis providing important information on the predictive value of different genetic subtypes of GIST. $K I T$-negative gastrointestinal stromal tumours: proof of concept and therapeutic implications. Am. J. Surg. Pathol. 28(7), 889-894 (2004). Initial and late resistance to imatinib in advanced gastrointestinal stromal tumours are predicted by different prognostic factors: a European Organisation for Research and Treatment of Cancer-Italian Sarcoma Group-Australasian Gastrointestinal Trials Group study. J. Clin. Oncol. 23(24), 5795-5804 (2005).

57 Corless CL, Schroeder A, Griffith D et al. PDGFRA mutations in gastrointestinal stromal tumours: frequency, spectrum and in vitro sensitivity to imatinib. J. Clin. Oncol. 23(23), 5357-5364 (2005).

58 Farag S, Somaiah N, Choi H et al. Clinical characteristics and treatment outcome in a large multicenter observational cohort of PDGFRA exon 18 mutated gastrointestinal stromal tumour (GIST) patients. J. Clin. Oncol. 34(Suppl.), Abstract 11011 (2016).

- Provides additional new information on the predictive implications of different PDGFRA mutations in GIST, potentially practice changing.

59 Joensuu H, Eriksson M, Sundby K et al. One vs three years of adjuvant imatinib for operable gastrointestinal stromal tumour: a randomized trial. JAMA 307(12), 1265-1272 (2012).

60 Mussi C, Schildhaus HU, Gronchi A et al. Therapeutic consequences from molecular biology for gastrointestinal stromal tumour patients affected by neurofibromatosis type 1 . Clin. Cancer Res. 14(14), 4550-4555 (2008).

61 Rege TA, Wagner AJ, Corless CL et al. 'Pediatric-type' gastrointestinal stromal tumours in adults: distinctive histology predicts genotype and clinical behaviour. Am. J. Surg. Pathol. 35, 495-504 (2011).

62 Heinrich MC, Corless CL, Blanke CD et al. Molecular correlates of imatinib resistance in gastrointestinal stromal tumours. J. Clin. Oncol. 24(29), 4764-4774 (2006).

63 Agaram NP, Wong GC, Guo T et al. Novel V600E $B R A F$ mutations in imatinib-naive and imatinib-resistant gastrointestinal stromal tumours. Genes Chromosomes Cancer 47(10), 853-859 (2008).

64 Gramza AW, Corless CL, Heinrich MC. Resistance to tyrosine kinase inhibitors in gastrointestinal stromal tumours. Clin. Cancer Res. 15(24), 7510-7518 (2009).

65 Liegl B, Kepten I, Le C, Zhu M et al. Heterogeneity of kinase inhibitor resistance mechanisms in GIST. J. Pathol. 216(1), 64-74 (2008).

66 Wardelmann E, Merkelbach-Bruse S, Pauls K et al. Polyclonal evolution of multiple secondary KIT mutations in gastrointestinal stromal tumours under treatment with imatinib mesylate. Clin. Cancer Res. 12(6), 1743-1749 (2006).

67 Heinrich MC, Maki RG, Corless CL et al. Primary and secondary kinase genotypes correlate with the biological and clinical activity of sunitinib in imatinib-resistant gastrointestinal stromal tumour. J. Clin. Oncol. 26(33), 5352-5359 (2008).

68 Miselli FC, Casieri P, Negri T et al. c-Kit/PDGFRA gene status alterations possibly related to primary imatinib resistance in gastrointestinal stromal tumours. Clin. Cancer Res. 13(8), 2369-2377 (2007).

69 Lasota J, Wang Z, Kim SY et al. Expression of the receptor for type I insulin-like growth factor (IGF1R) in gastrointestinal stromal tumours: an immunohistochemical study of 1078 cases with diagnostic and therapeutic implications. Am. J. Surg. Pathol. 37(1), 114-119 (2013).

70 Demetri GD, van Oosterom AT, Garrett CR et al. Efficacy and safety of sunitinib in patients with advanced gastrointestinal stromal tumour after failure of imatinib: a randomised controlled trial. Lancet 368(9544), 1329-1338 (2006).

71 Demetri GD, Reichardt P, Kang Y et al. Efficacy and safety of regorafenib for advanced gastrointestinal stromal tumours after failure of imatinib and sunitinib (GRID): an international, multicentre, randomised, placebo-controlled, Phase 3 trial. Lancet 381, 295-302 (2013).

72 Reichardt P, Demetri GD, Gelderblom H et al. Correlation of KIT and PDGFRA mutational status with clinical benefit in patients with gastrointestinal stromal tumour treated with sunitinib in a worldwide treatment-use trial. BMC Cancer 16(1), 22 (2016).

73 Prenen H, Cools J, Mentens N et al. Efficacy of the kinase inhibitor SU11248 against gastrointestinal stromal tumour mutants refractory to imatinib mesylate. Clin. Cancer Res. 12(8), 2622-2627 (2006).

74 Kollàr A, Maruzzo M, Messiou C et al. Regorafenib treatment for advanced, refractory gastrointestinal stromal tumour: a report of the UK managed access program. Clin. Sarcoma Res. 4, 17 (2014). 